\title{
Decomposing infinite 2-connected graphs into 3-connected components
}

\author{
R. Bruce Richter* \\ Department of Combinatorics and Optimization \\ University of Waterloo \\ Waterloo, Ont., Canada \\ brichter@math.uwaterloo.ca
}

Submitted: Mar 12, 2002; Accepted: Mar 16, 2004; Published: Mar 25, 2004

MR Subject Classifications: 05Cxx

\begin{abstract}
In the 1960's, Tutte presented a decomposition of a 2-connected finite graph into 3 -connected graphs, cycles and bonds. This decomposition has been used to reduce problems on 2-connected graphs to problems on 3-connected graphs. Motivated by a problem concerning accumulation points of infinite planar graphs, we generalize Tutte's decomposition to include all infinite 2-connected graphs.
\end{abstract}

\section{Introduction}

In [5], Tutte presents a decomposition of a finite 2-connected graph into 3-connected graphs, cycles and bonds. This is useful in turning problems about 2-connected graphs into problems about 3-connected graphs (see [4] for an example in which the full decomposition is used).

The following problem concerning accumulation points of infinite graphs embedded in the plane provides motivation for generalizing Tutte's decomposition to infinite graphs.

Question 1 Which (locally finite) planar graphs have embeddings in the plane with only finitely many accumulation points?

An answer to this question can be given in terms of forbidden subgraphs. One step in proving that these are all the forbidden subgraphs is the following technical lemma.

${ }^{*}$ The financial support of NSERC is acknowledged. 
Lemma 2 Let $G$ be a locally finite connected graph having two embeddings in the plane, one embedding having only finitely many accumulation points and the other having a specified finite set of vertices on the boundary of the same face, in a specific cyclic order. Then $G$ has an embedding in the plane having only finitely many accumulation points and having the specified finite set of vertices on the boundary of the same face, in the specific cyclic order.

One way of approaching this technical lemma is to first prove it for 3-connected graphs (the proof is easier in this case because both embeddings extend the same embeddings of the finite 3-connected subgraphs of $G$ ). It should then be possible to prove it for 2-connected graphs using the results of this work, and finally connected graphs should follow just from the block decomposition. Because induction is not readily available as a tool, it seems that one needs the entire Tutte decomposition to do the 2-connected case (and similarly the entire block decomposition to do the connected case).

We note that Droms, Servatius and Servatius [2] have actually proved our main result when restricted to locally finite graphs (which is in principle all that is needed for the technical lemma above). They were also interested in characterizing when a decomposition was of a locally finite graph. However, it seems that little extra work beyond Tutte's original arguments is required to provide the decomposition in general, and we do this here, in a completely self-contained way. But, as another example, one can prove Halin's forbidden subgraph characterization of countable connected graphs that have an embedding in the plane with no accumulation points [3] using the full force of Tutte's decomposition to reduce the problem to 3-connected graphs. (This is not necessarily recommended; Halin's proof is more elegant than this.)

It is also true that any set of non-crossing cuts provides a tree-decomposition of a graph (see [1] for the definition of a tree-decomposition; it is not required here). The hinges that are central to Tutte's theory are non-crossing 2-cuts and so there is a corresponding treedecomposition. However, the precise nature of the parts in the tree-decomposition is not clear just from these general considerations.

\section{Separations, Blocks and Hinges}

In this section, we introduce the notions of separations and hinges. This section is in very large measure extracted from [5] (with somewhat different notation and terminology adjusted to suit the context). In this work, a graph may be either finite or infinite, with no restrictions on size and it may have loops and multiple edges. A bond is a graph consisting of just two vertices and at least one edge, such that every edge has both vertices as its ends. A bond is a $k$-bond if it has precisely $k$ edges. Of necessity we allow infinite bonds in this work.

A separation in a graph $G$ is a pair $(H, K)$ of edge-disjoint non-null subgraphs $H$ and $K$ of $G$ such that $G=H \cup K$. For a non-negative integer $k$, a separation $(H, K)$ is a $k$-separation if $|E(H)| \geq k,|E(K)| \geq k$, and $|V(H \cap K)|=k$. The graph $G$ is $k$-connected if there is no $m$-separation, for all $m \in\{0,1, \ldots, k-1\}$. 
Note that if $G$ has fewer than $2 k$ edges, then $G$ has no $k$-separation. In particular, $K_{3}$ and the 3 -bond are $k$-connected for every positive integer $k$.

The definition given above says that $G$ is 1 -connected if and only if there is no 0separation. By considering, for a particular vertex $x$, the set of vertices reachable from $x$ by paths, we see that $G$ is connected if and only if $G$ is 1-connected.

Define the relation $\sim$ on the edges of $G$ by $e \sim f$ if either $e=f$ or $e$ and $f$ are in a cycle of $G$. It is a standard exercise to show that $\sim$ is an equivalence relation. A block of $G$ is either an isolated vertex or the subgraph of $G$ induced by an equivalence class of $\sim$. For finite graphs, this is the same as the maximal connected subgraphs of $G$ that have no cut-vertex (as long as a vertex incident with a loop and at least one other edge is a cut-vertex). It is easy to see that two blocks have at most one vertex in common. Furthermore, let $\mathcal{T}_{1}(G)$ denote the graph whose vertices are the blocks of $G$ and the cutvertices of $G$. A block $B$ is adjacent in $\mathcal{T}_{1}(G)$ to a cut-vertex $v$ if $v \in V(B)$; these are the only adjacencies in $\mathcal{T}_{1}(G)$. It is well-known (and easy to prove, even for infinite graphs) that if $G$ is connected, then $\mathcal{T}_{1}(G)$ is a tree.

Our goal is to provide an analog of $\mathcal{T}_{1}(G)$ when $G$ is 2-connected and the "blocks" are 3-connected. If $x, y$ are distinct vertices of a graph $G$, then $G+x y$ denotes the graph obtained from $G$ by adding an edge joining $x$ and $y$, even if there already is such an edge, while $[x, y]$ denotes the edgeless subgraph of $G$ consisting just of the vertices $x$ and $y$. The following result is a straightforward application of the definitions.

Lemma 3 Let $G$ be a 2-connected graph, let $(H, K)$ be a 2-separation of $G$, and let $[x, y]=H \cap K$. Then both $H+x y$ and $K+x y$ are 2-connected.

Let $G$ be a graph and let $H$ be a subgraph of $G$. A subgraph $K$ of $G$ is an $H$-bridge in $G$ if either $K$ is a 1-bond, both of whose vertices are in $H$ but whose edge is not in $H$, in which case $K$ is trivial, or $K$ is obtained from a component $L$ of $G-V(H)$ by adding to $L$ all the edges of $G$ that have at least one end in $L$ (together with all ends of these edges).

A hinge-separation of $G$ is a 2-separation $(H, K)$ of $G$ such that at least one of $H$ and $K$ is an $(H \cap K)$-bridge in $G$ and at least one of $H$ and $K$ is 2-connected. If $(H, K)$ is a hinge-separation, then $H \cap K$ is a hinge. Note that if $H$, say, is an $(H \cap K)$-bridge, then $|E(H)| \geq 2$ because $(H, K)$ is a 2 -separation and, therefore, $H$ is not a 1 -bond. In particular, $H$ has a vertex not in $H \cap K$. We have the following easy observation.

Lemma 4 Let $(H, K)$ be a 2-separation of a 2-connected graph $G$ and let $[x, y]=H \cap K$. Then $[x, y]$ is a hinge of $G$ if and only if either there are at least three $[x, y]$-bridges in $G$, at least one of which is not a 1-bond, or there are exactly two $[x, y]$-bridges, at least one of which is 2-connected.

The main point of this section are the following two results. The first proves that hinges do not cross, while the second characterizes 2-connected graphs with no hinge.

Lemma 5 Let $[x, y]$ be a hinge of a 2-connected graph $G$ and let $(H, K)$ be a 2-separation of $G$. Then there is an $[x, y]$-bridge $B$ of $G$ such that $H \cap K \subseteq B$. 
Proof: Let $[u, v]=H \cap K$ and suppose by way of contradiction there are distinct $[x, y]$-bridges $B_{u}$ and $B_{v}$ such that $u \in V\left(B_{u}\right) \backslash V\left(B_{v}\right)$ and $v \in V\left(B_{v}\right) \backslash V\left(B_{u}\right)$. As $x, y \in V\left(B_{u}\right) \cap V\left(B_{v}\right)$, we see that $\{x, y\} \cap\{u, v\}=\emptyset$.

Since $B_{u}$ contains the edges of $G$ incident with $u,\left|E\left(B_{u}\right)\right| \geq 2$. Likewise, $\left|E\left(B_{v}\right)\right| \geq 2$ so that, letting $\overline{B_{u}}$ denote the union of all the $[x, y]$-bridges except $B_{u},\left(B_{u}, \overline{B_{u}}\right)$ is a 2-separation of $G$. It follows from Lemma 4 that $\left(B_{u}, \overline{B_{u}}\right)$ is a hinge-separation of $G$.

For any vertex $w \in V(G) \backslash\{x, y\}$, there is a path $P$ in $G$ from $x$ to $y$ through $w$. There is a single $[x, y]$-bridge containing $P$, so at most one of $u$ and $v$ is in $P$ and, therefore, there is a path in $G$ joining $w$ to one of $x$ and $y$ that is disjoint from $u$ and $v$, except possibly for the $w$-end, if it is one of $u$ and $v$.

It follows that there are at most two non-trivial $[u, v]$-bridges in $G$. If there are two, then $x$ and $y$ are in different ones. As $u$ and $v$ are in different $[x, y]$-bridges in $G$, there is no edge of $G$ joining $u$ and $v$, so there are no trivial $[u, v]$-bridges. Since $(H, K)$ is a 2-separation of $G$ and $[u, v]=H \cap K$, there are exactly two [u,v]-bridges, one containing $x$ and the other containing $y$.

Note that this implies that there are exactly two $[x, y]$-bridges in $G$, since otherwise there is a path in $G$ joining $x$ and $y$ that is disjoint from $u$ and $v$. These bridges are just $B_{u}$ and $B_{v}$. We show that neither $B_{u}$ nor $B_{v}$ is 2-connected, contradicting the fact that $[x, y]$ is a hinge. Since the argument is the same in both cases, we deal only with $B_{u}$.

Let $X$ denote the vertices $w$ of $B_{u}$ for which there is a path $P$ from $w$ to $x$ such that $u$ is not an internal vertex of $P$. Similarly let $Y$ denote the vertices of $B_{u}$ for which $P$ goes from $w$ to $y$ without having $u$ as an internal vertex. Then the earlier remarks imply that $X \cup Y=V\left(B_{u}\right)$ and $X \cap Y=\{u\}$. For $z \in\{x, y\}$, let $L_{z}$ be the subgraph of $B_{u}$ induced by $Z$. Clearly, $\left(L_{x}, L_{y}\right)$ is a 1 -separation of $B_{u}$, as required.

Theorem 6 Let $G$ be a 2-connected graph with no hinge. Then $G$ is either 3-connected, a cycle, or a bond.

Proof: Suppose $G$ is neither 3-connected nor a bond. We show $G$ is a polygon. Since $G$ is not 3-connected, there is a 2 -separation $(H, K)$ of $G$. Let $[x, y]=H \cap K$. Since $G$ is not a bond, there is a non-trivial $[x, y]$-bridge. Since $[x, y]$ is not a hinge, Lemma 4 implies there are exactly two $[x, y]$-bridges and both are non-trivial. Thus, $H$ and $K$ are the two $[x, y]$-bridges and neither is 2-connected. We prove that $H$ and $K$ are both paths having $x$ and $y$ as their ends. Since the argument is the same for both, we deal only with $H$.

Lemma 3 implies $H+x y$ is 2-connected, so if $(L, M)$ is any 1-separation of $H$, we may choose the labelling so that $x \in V(L) \backslash V(M)$ and $y \in V(M) \backslash V(L)$. Let $P$ be any path in $H$ from $x$ to $y$ and let $B_{1}, B_{2}, \ldots, B_{r}$ be the blocks of $H$ containing the edges of $P$. There are no other blocks of $H$.

If any $B_{i}$ has two or more edges, then let $u, v$ be the attachments of $B_{i}$ in $G$. There is a $[u, v]$-bridge $B$ in $G$ containing $K$. We see that $(B, \bar{B})$ is a hinge-separation in $G$, a contradiction. Hence each $B_{i}$ has exactly one edge, so $H=P$, as claimed. 


\section{Cleavage Units}

In this section we define the cleavage units of a graph. The main point is to show that cleavage units are either 3-connected, a cycle, or a bond. Let $G$ be a 2-connected graph and let $[x, y]$ be a hinge of $G$. Let $\mathcal{B}_{[x, y]}$ denote the set of $[x, y]$-bridges in $G$ and let $\mathcal{B}_{[x, y]}^{2}$ denote the set of non-trivial $[x, y]$-bridges in $G$. For each $B \in \mathcal{B}_{[x, y]}^{2}$, we introduce a new edge $e_{B}^{1}$ having $x$ and $y$ as its ends. If $\left|\mathcal{B}_{[x, y]}\right| \geq 3$, then we also introduce, for each $B \in \mathcal{B}_{[x, y]}^{2}$, a second new edge $e_{B}^{2}$, also having $x$ and $y$ as its ends. These new edges are virtual and the set of them is $\operatorname{vir}(G)$. Thus, every hinge of $G$ introduces at least two virtual edges. Let $\hat{G}$ denote the graph $(V(G), E(G) \cup \operatorname{vir}(G))$. For a non-trivial bridge $B$ of a hinge $[x, y]$ of $G$, set $\hat{B}$ to be the graph obtained from $B$ by adding any virtual edge that either has an end in $B$ different from both $x$ and $y$ or is $e_{B}^{1}$.

Define the relation $\approx$ on $E(\hat{G})$ by $e \approx f$ if, for every hinge $[x, y]$ of $G$ and every non-trivial $[x, y]$-bridge $B$ in $G$, either both $e$ and $f$ are in $\hat{B}$ or both are not in $\hat{B}$. It is a complete triviality that $\approx$ is an equivalence relation. A cleavage unit of $G$ is any subgraph of $\hat{G}$ induced by an equivalence class of $\approx$. If $e$ and $f$ are edges of $\hat{G}$ in different cleavage units, then there is a hinge $[x, y]$ and an $[x, y]$-bridge $B$ such that one of $e$ and $f$ is in $E(\hat{B})$ and the other is not. Such a hinge separates $e$ and $f$. The following easy lemma describes one type of cleavage unit and is useful later.

Lemma 7 Let $G$ be a 2-connected graph and let $[x, y]$ be a hinge of $G$. If $\left|\mathcal{B}_{[x, y]}\right| \geq 3$, then the set of edges of $G$ having $x$ and $y$ as ends, together with the edges of $\hat{G}$ of the form $e_{B}^{2}$, for $B \in \mathcal{B}_{[x, y]}^{2}$, induces a cleavage unit of $G$. Furthermore, this cleavage unit is $a\left|\mathcal{B}_{[x, y]}\right|$-bond.

We denote by $K_{[x, y]}$ the cleavage unit described in Lemma 7 .

We are interested in proving that the cleavage units give a tree-like decomposition of $G$. To this end, the decomposition graph of a 2-connected graph $G$ is the graph $\mathcal{T}(G)$ whose vertices are the cleavage units of $G$ and two cleavage units $K_{1}, K_{2}$ are adjacent in $\mathcal{T}(G)$ if there is a hinge $[x, y]$ of $G$ and either there is an $[x, y]$-bridge $B$ such that $e_{B}^{1}$ is in one of $K_{1}$ and $K_{2}$, while $e_{B}^{2}$ is in the other, or there are just two $[x, y]$-bridges $B$ and $B^{\prime}$, and $e_{B}^{1}$ in one of $K_{1}$ and $K_{2}$ while $e_{B^{\prime}}^{1}$ is in the other. Note that if $G$ has no hinge, then $G$ itself is the only cleavage unit.

Lemma 8 Let $G$ be a 2-connected graph and let $[x, y]$ be a hinge of $G$. Let $u$ and $v$ be distinct vertices of $G$ such that $\{u, v\} \neq\{x, y\}$. Then $[u, v]$ is a hinge of $G$ if and only if there is an $[x, y]$-bridge $B$ in $G$ such that $[u, v]$ is a hinge of $B+e_{B}^{1}$.

Proof: Suppose first that $[u, v]$ is a hinge of $G$. Lemma 5 implies there is an $[x, y]$-bridge $B$ in $G$ such that $[u, v] \subseteq B$. Set $H=B+e_{B}^{1}$.

Let $J_{G}$ be the $[u, v]$-bridge in $G$ containing $[x, y]$ and let $J_{B}=(J \cap B)+e_{B}^{1}$. As at least one of $x$ and $y$ is not in $\{u, v\},\left|E\left(J_{B}\right)\right| \geq 2$. Since $[u, v]$ is a hinge of $G$, the union $K$ of the $[u, v]$-bridges in $G$ other than $J_{G}$ has at least two edges. Thus $\left(J_{B}, K\right)$ is a 
2-separation of $H$. Moreover, either $J_{G}$ or $K$ is 2-connected, which implies that either $J_{B}$ or $K$ is 2-connected, i.e., $[u, v]$ is a hinge of $H$.

Conversely, suppose $[u, v]$ is a hinge of $H=B+e_{B}^{1}$. Let $J_{B}$ denote the $[u, v]$-bridge in $H$ containing $e_{B}^{1}$ and let $J_{G}$ denote the union of $J_{B}-e_{B}^{1}$ with all the $[x, y]$-bridges in $G$ other than $B$. Letting $K$ denote the union of the $[u, v]$-bridges in $B$ other than $J_{B}$, we see (as above) that $\left(J_{G}, K\right)$ is a hinge-separation of $G$.

The following straightforward corollary is the key to proving that $\mathcal{T}(G)$ is a tree.

Corollary 9 Let $G$ be a 2-connected graph, let $[x, y]$ be a hinge of $G$, and let $K$ be a cleavage unit of $G$. Then either $K=K_{[x, y]}$ or there is a non-trivial $[x, y]$-bridge $B$ such that $K$ is a cleavage unit of $B+e_{B}^{1}$.

We conclude this section by showing that no cleavage unit has a hinge. In the finite case, this follows by an easy induction based on Corollary 9 .

Theorem 10 Let $G$ be a 2-connected graph and let $K$ be a cleavage unit of $G$. Then $K$ is either 3-connected, a cycle, or a bond.

Proof: By Theorem 6, it suffices to show that $K$ has no hinge-separation. Suppose to the contrary that $(H, J)$ is a hinge-separation of $K$. Let $[x, y]=H \cap J$ and choose the labelling so that $H$ is an $[x, y]$-bridge in $K$. We construct a subgraph $H^{\prime}$ of $G$ as follows. Delete any $e \in E(H)$ having $x$ and $y$ as its ends (there is at most one such). For every hinge $[u, v]$ of $G$ such that $[u, v] \subseteq H$ and $\{u, v\} \neq\{x, y\}$, there is a unique $[u, v]$-bridge $B$ in $G$ that contains $J \cap G$. Replace $e=e_{B}^{1}$ in $H$ with the union of all the $[u, v]$-bridges in $G$ other than $B$. Clearly the only attachments of $H^{\prime}$ in $G$ are $x$ and $y$ and $H^{\prime}$ is an $[x, y]$-bridge. Let $J^{\prime}$ denote the union of the remaining $[x, y]$-bridges in $G$. Since $(H, J)$ is a hinge-separation of $K$, one of $H$ and $J$ is 2-connected. It is straightforward to show the corresponding one of $H^{\prime}$ and $J^{\prime}$ is also 2-connected, so that $\left(H^{\prime}, J^{\prime}\right)$ is a hinge-separation of $G$. But then every edge of $H$ is separated by $[x, y]$ in $G$ from every edge in $J$, a contradiction.

\section{The decomposition tree}

In this section we prove that the graph $\mathcal{T}(G)$ is a tree. This is done in two parts, the first showing that $\mathcal{T}(G)$ is connected and the second showing that every edge is an isthmus. In fact, the second is a simple consequence of the first and Corollary 9.

Theorem 11 Let $G$ be a 2-connected graph. Then $\mathcal{T}(G)$ is connected.

Proof: We show that if $e$ and $f$ are any two edges of $\hat{G}$, then there is a path in $\mathcal{T}(G)$ joining the two cleavage units $K_{e}$ and $K_{f}$ containing $e$ and $f$, respectively. It is clearly sufficient to prove the result in the case $e$ and $f$ have an end $x$ in common. 
Let $y$ and $z$ be the other ends of $e$ and $f$, respectively. Let $Q$ be a path in $\hat{G}-x$ joining $y$ and $z$. We proceed by induction on the length $n$ of $Q$, the base being the case $n=0$, which follows from Lemma 7 . Thus, we assume $y \neq z$.

If there is no hinge separating $e$ from $f$, then they are in the same cleavage unit and the result is trivial. Thus, we may assume there is a hinge separating $e$ from $f$. Evidently, such a hinge is of the form $[x, w]$ and $w$ is a vertex of $Q$. If $w \notin\{y, z\}$, then the result is an easy application of the inductive assumption. So we can suppose the only possible hinges separating $e$ from $f$ are $[x, y]$ and $[x, z]$. Choose the labelling so that $[x, y]$ is such a hinge.

Let $B$ be the $[x, y]$-bridge in $G$ containing $z$. Let $K$ be the cleavage unit of $G$ containing $e_{B}^{1}$. Evidently $K$ is connected in $\mathcal{T}(G)$ by a path of length at most two to $K_{e}$. If $[x, z]$ is not a hinge, then $f$ and $e_{B}^{1}$ are in the same cleavage unit and we are done. Otherwise, let $B^{\prime}$ be the $[x, z]$-bridge in $G$ containing $y$. Then $K$ is also the cleavage unit of $G$ containing $e_{B^{\prime}}^{1}$ and $K$ is joined to $K_{f}$ by a path of length at most two. Thus, $K_{e}$ and $K_{f}$ are connected by a path in $\mathcal{T}(G)$, as claimed.

Theorem 12 Let $G$ be a 2-connected graph. Then either $G$ has no hinge or $\mathcal{T}(G)$ is a tree.

Proof: It suffices to prove that every edge of $\mathcal{T}(G)$ is an isthmus. Corollary 9 shows that if $[x, y]$ is any hinge of $G$, then the cleavage units of $G$ are precisely the cleavage units of the graphs $B+e_{B}^{1}$, for $B \in \mathcal{B}_{[x, y]}^{2}$, plus, if $\left|\mathcal{B}_{[x, y]}\right| \geq 3$, the bond cleavage unit $K_{[x, y]}$.

The edge $e_{B}^{1}$ (after identification) separates in $\mathcal{T}(G)$ all the cleavage units of $B+e_{B}^{1}$ from all the cleavage units of $G$ contained in other bridges.

\section{Uniquess of the decomposition}

In this section we prove there is only one decomposition of a 2-connected graph into 3connected graphs, cycles, and bonds. As stated, it is not true, since, for example, a long cycle may be interpreted as having many different decompositions. Taking our cue from [2], we provide a little more detail about the nature of the decomposition. Let $\mathcal{T}$ be a tree and let $\mathcal{G}$ be an assignment of a 2-connected hinge-free graph $\mathcal{G}(t)$ to each vertex $t$ of $\mathcal{T}$.

Suppose that, for every vertex $t$ of $\mathcal{T}$, there is an injection $f_{t}$ from the edges of $\mathcal{T}$ incident with $t$ to the edges of $\mathcal{G}(t)$. Let $\mathcal{F}$ denote the collection of injections $f_{t}$. The triple $(\mathcal{T}, \mathcal{G}, \mathcal{F})$ is an amalgamtion tree. The graph $G(\mathcal{T}, \mathcal{G}, \mathcal{F})$ is obtained by identifying, for every edge $t t^{\prime}$ of $\mathcal{T}$, the ends of the edges $f_{t}\left(t t^{\prime}\right)$ and $f_{t^{\prime}}\left(t t^{\prime}\right)$ and deleting $f_{t}\left(t t^{\prime}\right)$ and $f_{t^{\prime}}\left(t t^{\prime}\right)$. We assume that $f_{t}\left(t t^{\prime}\right)$ and $f_{t^{\prime}}\left(t t^{\prime}\right)$ determine which of the two possible identifications is to be used.

Following [2], a 3-block decomposition of a graph $G$ is an amalgamation tree $(\mathcal{T}, \mathcal{G}, \mathcal{F})$ such that: 
1. $G=G(\mathcal{T}, \mathcal{G}, \mathcal{F})$;

2. if $t t^{\prime} \in E(\mathcal{T})$, then $\mathcal{G}(t)$ and $\mathcal{G}\left(t^{\prime}\right)$ are not both bonds and are not both polygons; and

3. for each edge $t t^{\prime}$ of $\mathcal{T}$, if $\mathcal{T}^{\prime}$ denotes the component of $\mathcal{T}-t t^{\prime}$ containing $t$, there is a path in $G\left(\mathcal{T}^{\prime}, \mathcal{G}^{\prime}, \mathcal{F}^{\prime}\right)-f_{t}\left(t t^{\prime}\right)$ joining the ends of $f_{t}\left(t t^{\prime}\right)$, where $\mathcal{G}^{\prime}$ and $\mathcal{F}^{\prime}$ are the restrictions of $\mathcal{G}$ and $\mathcal{F}$, respectively, to $\mathcal{T}^{\prime}$.

Condition 3 ensures that the graph $G(\mathcal{T}, \mathcal{G}, \mathcal{F})$ is 2 -connected. Condition 2 is the one that drives the uniqueness. Without it, bonds and cycles would not have unique decompositions.

We remark that in [2] there are two additional considerations required to ensure local finiteness of $G: \mathcal{G}(t)$ is locally finite; and a finiteness condition analogous to (2) to ensure that a vertex of $G(\mathcal{T}, \mathcal{G}, \mathcal{F})$ has finite degree. We are not restricting our attention to locally finite graphs and therefore have no need of this condition.

Let $G$ be a 2-connected graph. If $\mathcal{K}(G)$ denotes the set of cleavage units of $G$, the identity map gives a hinge-free assignment of the cleavage units to the vertices of $\mathcal{T}(G)$. We shall refer to this map also as $\mathcal{K}(G)$. Likewise, every edge $t t^{\prime}$ of $\mathcal{T}(G)$ is obtained by the identification of an edge of the cleavage unit $t$ of $G$ with the cleavage unit $t^{\prime}$ of $G$. Let $f_{t}\left(t t^{\prime}\right)$ be the identified edge of $t$ and let $\mathcal{F}(G)$ be the collection of these functions $f_{t}$.

Theorem 13 Let $G$ be a 2-connected graph. Then $(\mathcal{T}(G), \mathcal{K}(G), \mathcal{F}(G))$ is a 3-block decomposition of $G$.

In order to prove this result, we will use the following fact.

Lemma 14 Let $G$ be a 2-connected graph and let $K$ be a cleavage unit of $G$ that is a polygon. If $[x, y]$ is any hinge of $G$ such that $[x, y] \subseteq K$, then $x$ and $y$ are adjacent in $K$. Furthermore, if $B$ is the $[x, y]$-bridge in $G$ such that $K$ is a cleavage unit of $B+e_{B}^{1}$, then every vertex of $K-\{x, y\}$ is a cut-vertex of $B$.

Proof: If $x$ and $y$ are not adjacent in $K$, then the two $[x, y]$-bridges in $K$ are separated by $[x, y]$ in $G$, contradicting the fact that $K$ is a cleavage unit of $G$.

By the preceding paragraph, if $[u, v]$ is any hinge of $G$ such that $[u, v] \subseteq K$, then $u$ and $v$ are adjacent in $K$. Each edge $u v$ of $K$ different from $x y$ is either an edge of $G$ or of the form $e_{B_{[u, v]}}^{1}$, where $B_{[u, v]}$ is the unique $[u, v]$-bridge in $G$ containing both $x$ and $y$. For $u \notin\{x, y\}$, let $P$ be the path in $K$ from $x$ to $u$ and let $Q$ be the path in $K$ from $y$ to $u$. For any edge $a b$ of $P$, let $H$ denote the union of all the $[a, b]$-bridges in $G$ other than $B_{[a, b]}$. Obtain $J$ in the same way from all the edges of $Q$. Then $(H, J)$ is a 1-separation of $B$ and $[u]=H \cap J$.

Proof of Theorem 13: For Condition (2), cleavage units of type $K_{[x, y]}$ are clearly not adjacent. If $K$ is a polygon cleavage unit adjacent to the cleavage unit $J$ in $\mathcal{T}(G)$, then let $[x, y]$ be the hinge separating $K$ and $J$. There must be exactly two $[x, y]$-bridges in $G$. 
Label them $B_{K}$ and $B_{J}$ so that $K$ is a cleavage unit of $B_{K}+e_{B_{K}}^{1}$ and $J$ is a cleavage unit of $B_{J}+e_{B_{K}}^{1}$. By Lemma 14, every vertex of $K-\{x, y\}$ is a cut-vertex of $B_{K}$, so $B_{K}$ is not 2 -connected. Therefore, $B_{J}$ is 2 -connected and by Lemma $14 J$ is not a polygon.

As for Condition (3), this is an immediate consequence of the fact that if $x y=f_{t}\left(t t^{\prime}\right)$, then $[x, y]$ is a hinge and some $[x, y]$-bridge in $G$ is contained in $G\left(\mathcal{T}^{\prime}\right)$.

What remains to be proved is the uniqueness.

Theorem 15 Let $G$ be a 2-connected graph and let $(\mathcal{T}, \mathcal{G}, \mathcal{F})$ be a 3-block decomposition of $G$. Then $(\mathcal{T}, \mathcal{G}, \mathcal{F})=(\mathcal{T}(G), \mathcal{K}(G), \mathcal{F}(G))$.

Proof: Let $t t^{\prime} \in E(\mathcal{T})$ and let $\mathcal{T}_{t}$ and $\mathcal{T}_{t^{\prime}}$ be the components of $\mathcal{T}-t t^{\prime}$ containing $t$ and $t^{\prime}$, respectively. Let $H=G\left(\mathcal{T}_{t}\right)-f_{t}\left(t t^{\prime}\right)$ and let $K=G\left(\mathcal{T}_{t^{\prime}}\right)-f_{t^{\prime}}\left(t t^{\prime}\right)$. Evidently, $(H, K)$ is a 2-separation of $G$. We claim that $(H, K)$ is a hinge-separation.

If either of the graphs $\mathcal{G}(t)-f_{t}\left(t t^{\prime}\right)$ and $\mathcal{G}\left(t^{\prime}\right)-f_{t^{\prime}}\left(t t^{\prime}\right)$ is 2-connected, then the corresponding one of $H$ and $K$ is 2-connected, by Condition (3). As $\mathcal{G}(t)-f_{t}\left(t t^{\prime}\right)$ is 2-connected unless $\mathcal{G}(t)$ is a polygon, Condition (2) implies at least one of $H$ and $K$ is 2-connected.

We must show that one of $H$ and $K$ is an $(H \cap K)$-bridge in $G$. Suppose $H$ is not an $(H \cap K)$-bridge in $G$. We claim that $\mathcal{G}(t)$ is a bond. The same argument applies to $K$, so Condition (2) implies at least one of $H$ and $K$ is an $(H \cap K)$-bridge in $G$.

Let $[x, y]=H \cap K$. If every $[x, y]$-bridge in $H$ is a 1-bond, then trivially $\mathcal{G}(t)=$ $H+f_{t}\left(t t^{\prime}\right)$. Thus, we may assume there is a non-trivial $[x, y]$-bridge in $H$.

If $\mathcal{G}(t)$ is not a bond, then it has a non-trivial $[x, y]$-bridge $B$ in $\mathcal{G}(t)$. Let $B_{H}$ denote the $[x, y]$-bridge in $H$ containing the vertices of $B-\{x, y\}$. Then $B_{H}+f_{t}\left(t t^{\prime}\right)=G\left(\mathcal{T}^{\prime}\right)$ for some subtree $\mathcal{T}^{\prime}$ of $\mathcal{T}$. Since the only edge in $G\left(\mathcal{T}^{\prime}\right)$ of the form $f_{s}(e)$ is $f_{t}\left(t t^{\prime}\right)$, it must be that $\mathcal{T}^{\prime}=\mathcal{T}$. But there was another $[x, y]$-bridge in $H$, a contradiction.

Hence $\mathcal{G}(t)$ is a bond, as claimed.

Thus, we have shown that every 2-separation corresponding to an edge of $\mathcal{T}$ is a hingeseparation. Conversely, if $(H, K)$ is a hinge-separation, then we claim there is an edge of $\mathcal{T}$ giving this separation.

Let $[x, y]$ be a hinge of $G$, and suppose $[x, y]$ is not a hinge corresponding to any edge of $\mathcal{T}$. For any edge $e$ of $\mathcal{T}$, with components $\mathcal{T}^{\prime}$ and $\mathcal{T}^{\prime \prime}$ of $\mathcal{T}-e$, either $[x, y] \subseteq G\left(\mathcal{T}^{\prime}\right)$ or $[x, y] \subseteq G\left(\mathcal{T}^{\prime \prime}\right)$, but not both, since $[x, y]$ does not cross the hinge associated with $e$. Therefore, there is a unique vertex $t$ of $\mathcal{T}$ such that $[x, y] \subseteq \mathcal{G}(t)$. We claim that $[x, y]$ is a hinge of $\mathcal{G}(t)$.

This is clear if there are at least three $[x, y]$-bridges in $G$, since then there are three in $\mathcal{G}(t)$. If there are exactly two $[x, y]$-bridges in $G$, let $B$ be one of them that is 2connected. We will be done when we prove that $B_{t}$ is 2-connected. Suppose that $(H, K)$ is a separation of $B_{t}$ with $|V(H \cap K)| \leq 1$.

An easy argument shows that if $P$ is a path in $G$ that starts and ends in $\mathcal{G}(t)$ then there is a path $P_{t}$ in $\mathcal{G}(t)$, obtained from $P$ by replacing some subpaths by edges of the form $f_{t}(e)$. Since $B$ is 2-connected and an $x y$-bridge, there is a $\theta$-subgraph $J$ of $B$ containing $x$ and $y$, neither as a degree three vertex. This implies that there is a $\theta$-subgraph $J_{t}$ of 
$B_{t}$ containing $x$ and $y$, neither as a degree three vertex. Hence, $J_{t}$ is contained in either $H$ or $K$; choose the labelling so that $J_{t} \subseteq H$.

Because $\mathcal{G}(t)$ has no loops, $K$ must contain a vertex $w$ not in $H$. As $B$ is 2 -connected, there are paths $P$ and $Q$ in $B$ joining $w$ to $x$ and $y$, respectively, such that $P$ and $Q$ are disjoint except for $w$. Thus, the paths $P_{t}$ and $Q_{t}$ join $w$ to $x$ and $y$ in $B_{t}$ and are disjoint except for $w$. But $|V(H) \cap V(K)| \leq 1$, a contradiction. Hence $B_{t}$ is 2-connected. It follows that $[x, y]$ is a hinge of $\mathcal{G}(t)$, a contradiction.

Thus, every edge of $\mathcal{T}$ corresponds to a hinge and every hinge corresponds to at least one edge of $\mathcal{T}$. Let $e$ be an edge of $\mathcal{T}$ incident with the vertex $t$ and let $\mathcal{T}^{\prime}$ be the component of $\mathcal{T}$ containing $t$. Suppose $\mathcal{G}(t)$ is not a bond. We claim that if $x$ and $y$ are the ends of $f_{t}(e)$, then $G\left(\mathcal{T}^{\prime}\right)-f_{t}(e)$ is an $[x, y]$-bridge in $G$.

For any two vertices $u$ and $v$ of $G\left(\mathcal{T}^{\prime}\right)-\{x, y\}$, there is a $u v$-path in $G\left(\mathcal{T}^{\prime}\right)$ that is disjoint from $x$ and $y$. (There are two cases: if the graphs $\mathcal{G}\left(s_{u}\right)$ and $\mathcal{G}\left(s_{v}\right)$ containing $u$ and $v$ respectively are such that $s_{u}$ and $s_{v}$ are in the same component of $\mathcal{T}^{\prime}-t$, the result is easy; if not, then the edges $e_{u}$ and $e_{v}$ where their respective components attach to $t$ satisfy the property that $f_{t}\left(e_{u}\right)$ and $f_{t}\left(e_{v}\right)$ each has an end other than $x$ and $y$ and there is a path in $\mathcal{G}(t)-\{x, y\}$ joining these ends. Since there is no edge joining $x$ and $y$ in $G\left(\mathcal{T}^{\prime}\right)$ other than $f_{t}(e)$, the claim follows.

\section{References}

[1] Reinhard Diestel, Graph Theory, Springer-Verlag, Berlin, New York, 2000.

[2] Carl Droms, Brigitte Servatius, and Herman Servatius, The structure of locally finite two-connected graphs, Electron. J. Combin. 2 (1995), Research Paper 17.

[3] R. Halin, Zur häufungspunktfreien Darstellung abzählbarer Graphen in der Ebene, Arch. Math. (Basel) 17 (1966), 239-243.

[4] R.B. Richter, P.D. Seymour, and J. Širáň, Circular embeddings of planar graphs in nonspherical surfaces, Discrete Math. 126 (1994), 273-280.

[5] W.T. Tutte, Connectivity in graphs, Mathematical Expositions, No. 15 University of Toronto Press, Toronto, Ont.; Oxford University Press, London 1966 